\title{
Revisión de la proyección social institucional a partir del impacto formativo en graduados
}

\author{
F. Giraudo \\ Universidad Católica de Córdoba, Argentina \\ D. Gargantini
}

Universidad Católica de Córdoba, Argentina - Red de Responsabilidad Social Universitaria (RSU)- Asociación de Universidades confiadas a la Compañía de Jesús en América Latina (AUSJAL)

\section{Resumen}

Desde una noción de calidad educativa concebida como aquella que asegura la adquisición de conocimientos significativos y el desarrollo de capacidades ciudadanas comprometidas con la transformación de la realidad, las universidades confiadas a la Compañía de Jesús en América Latina han ido generando iniciativas que apuntan a la formación de los estudiantes para el compromiso y la solidaridad. Este artículo tiene como objetivo determinar los factores emergentes vinculados a la cultura institucional que inciden en la formación de sus graduados, y de esta manera, inferir los cambios a introducir en la política de proyección social institucional. La metodología empleada incluyó un enfoque analítico interpretativo, retrospectivo y transversal, desarrollado a través de un diseño metodológico cualitativo a través del análisis documental, entrevistas con referentes institucionales, graduados, y referentes externos (empleadores y representantes de entidades deontológicas). A la luz de los resultados fue posible determinar las revisiones valóricas, procedimentales e instrumentales que la política de proyección social de la universidad debiera encarar, a fin de cumplir en profundidad con la misión que le ha sido encomendada.

\section{Palabras clave}

Aprendizaje-servicio, impacto formativo, perfil profesional, política de proyección social. 


\title{
Review of the institutional social projection from the educational impact on graduates
}

\begin{abstract}
From a notion of educational quality conceived as one that ensures the acquisition of significant knowledge and the development of citizen capacities committed to the transformation of reality, the universities entrusted to the Society of Jesus in Latin America have been generating initiatives that aim at training of students for commitment and solidarity. This article aims to determine the emerging factors linked to the institutional culture that affect the training of its graduates, and in this way, infer the changes to be introduced in the policy of institutional social projection. The methodology used included an interpretive, retrospective and cross-sectional analytical approach, developed through a qualitative methodological design through documentary analysis, interviews with institutional referents, graduates, and external referents (employers and representatives of deontological entities). In light of the results, it was possible to determine the value, procedural and instrumental reviews that the university's social projection policy should face, in order to fully comply with the mission that has been entrusted to it.
\end{abstract}

\section{Key words}

Service-learning, training impact, professional profile, social projection policy. 


\title{
Revisió de la projecció social institucional a partir de I’impacte formatiu en graduats
}

\author{
Resum \\ Des d'una noció de qualitat educativa concebuda com aquella que assegura \\ l'adquisició de coneixements significatius i el desenvolupament de capacitats \\ ciutadanes compromeses amb la transformació de la realitat, les universitats \\ confiades a la Companyia de Jesús a I'Amèrica Llatina han generat iniciatives que \\ apunten a la formació dels estudiants per al compromís i la solidaritat. Aquest article \\ té com a objectiu determinar els factors emergents vinculats a la cultura institucional \\ que incideixen en la formació dels seus graduats, i així inferir els canvis a introduir en \\ la política de projecció social institucional. La metodologia emprada va incloure un \\ enfocament analític interpretatiu, retrospectiu i transversal, desenvolupat a través \\ d'un disseny metodològic qualitatiu a través de l'anàlisi documental, entrevistes amb \\ referents institucionals, graduats i referents externs (empleadors i representants \\ d'entitats deontològiques). A la llum dels resultats va ser possible determinar les \\ revisions valòriques, procedimentals i instrumentals que la política de projecció social \\ de la universitat ha d'encarar, per tal de complir en profunditat la missió que li ha \\ estat encomanada.
}

\section{Paraules clau}

Aprenentatge servei, impacte formatiu, perfil professional, política de projecció social. 


\section{Introducción}

La calidad educativa es un objetivo central de todo centro de educación superior. A pesar de los estándares nacionales e internacionales, todo concepto de calidad está asociado a un ideal a alcanzar, y no existe disociado de una misión particular.

Para las instituciones católicas, una institución educativa de excelencia es aquella que agrega el mayor valor al desarrollo y maduración integral de los estudiantes y de los actores involucrados. Esta educación integral supone un proceso que asegure la adquisición de conocimientos significativos, y el desarrollo de capacidades que permitan al estudiante concebirse inmerso en una realidad social de la que es parte activa y, frente a la cual se desempeña no sólo como experto en un ámbito específico, sino como ciudadano competente y comprometido con la transformación humanizadora y sostenible de su cultura (Papa Francisco, 20 de febrero del 2020).

La formación de talentos humanos para las tareas del cuidado y la transformación en búsqueda de un futuro esperanzador resulta así un objetivo central (Aguilar, 2021), por lo que una formación de calidad se encuentra asociada a: una investigación comprometida y pertinente (apostolado intelectual); pedagogías activas (protagonismo estudiantil); conocimiento significativo y relevante (articulación docencia-proyección social/aprendizaje-servicio); una ciudadanía activa (recuperación de la dimensión cívica-solidaridad e incidencia);y una reflexión crítica (pensamiento crítico-transformador). Frente a estos desafíos, las universidades jesuitas han realizado opciones para:

...incluir la promoción de la justicia, como expresión y servicio de la fe, modo de cuidar la creación, contenido del diálogo con otras religiones y motivación para la transformación de la cultura, (como forma de) ayudar a la propia Iglesia a transmitir su condición solidaria y compasiva y a hacer su mensaje más creíble para los no creyentes. Su educación formativa tiene un importante influjo en lo que los estudiantes -hombres y mujeresllegan a ser; la investigación que realiza posee la capacidad de desentrañar las causas estructurales que dan lugar a la injusticia y de sugerir propuestas que generen mejoras significativas para las personas desfavorecidas, pudiendo incluso ejercer la denuncia; y la propia institución universitaria se desenvuelve en un entorno de relaciones sociales donde su propia cultura interna y el modo de enfrentar la realidad tienen un fuerte influjo. (Álvarez, 2014, p. 15)

A partir de la adopción del paradigma de la Responsabilidad Social Universitaria (RSU), las universidades jesuitas en Latinoamérica promueven experiencias vivenciales de acercamiento a la realidad social, donde

Giraudo, F. y Gargantini, D. (2021). Revisión de la proyección social institucional a partir del impacto formativo en graduados. RIDAS, Revista Iberoamericana de Aprendizaje Servicio, 12, $104-114$. DOI10.1344/RIDAS2021.12.11 
el aprendizaje-servicio resulta una de las metodologías privilegiadas. Las mismas se desarrollan a partir de prácticas en proyectos sociales, que buscan incentivar las capacidades y competencias de los futuros profesionales frente a las problemáticas vigentes. Junto con ello se incentiva el compromiso creativo y constructivo desde un ejercicio ciudadano, democrático y de liderazgo ético en la construcción de una sociedad sostenible y más justa. La Responsabilidad Social Universitaria, como eje transversal, es concebida como:

...la habilidad y efectividad de la universidad para responder a las necesidades de transformación de la sociedad donde está inmersa, mediante el ejercicio de sus funciones sustantivas: docencia, investigación, extensión y gestión interna. Estas funciones deben estar animadas por la búsqueda de la promoción de la justicia, la solidaridad, la equidad social y el desarrollo humano sustentable. (Asociación de Universidades Confiadas a la Compañía de Jesús en América Latina [AUSJAL], 2014, p.15)

En la UCC, este tipo de experiencias se favorecen desde la política de proyección social (PPS) (Universidad Católica de Córdoba, 2011). La misma se sustenta en un modelo académico asociativo y multidisciplinario, comprometido con la cocreación e intercambio de saberes en íntima relación con los actores sociales.

A su amparo se han desarrollado numerosas iniciativas (proyectos bianuales de proyección social con vinculación curricular, proyectos especiales, proyectos de bienestar de la comunidad interna, programas estables de proyección social, proyectos de apoyo a la gestión, espacios de incidencia en políticas públicas y participación en redes). En todas ellas el aprendizaje-servicio posee un lugar privilegiado, por su aporte a la formación que se busca.

A partir de estos antecedentes y a fin de alcanzar la formación deseada, resulta importante evaluar el impacto de la PPS de la UCC (Argentina) en sus egresados. Este trabajo busca determinar los factores emergentes vinculados a la cultura-espiritualidad institucional en la Facultad de Ciencias Químicas, que inciden en la formación y el perfil de sus graduados, y de esta manera, inferir los cambios a introducir en la PPS y en las metodologías pedagógicas promovidas.

\section{Metodología ${ }^{1}$}

Durante 2018 a 2021 y bajo el formato de tesis doctoral, se propuso aportar herramientas metodológicas que permitieran correlacionar los procesos educativos de acuerdo con la PPS planteada en la universidad (con foco específico en la Facultad de Ciencias Químicas), con respecto a la formación y perfil logrados en sus graduados. Para ello se optó por seguir un enfoque analítico interpretativo, retrospectivo y transversal, desarrollado a través de un

${ }^{1}$ El presente apartado se encuentra elaborado a partir de los aportes de Giraudo y Gargantini (2019).

Giraudo, F. y Gargantini, D. (2021). Revisión de la proyección social institucional a partir del impacto formativo en graduados. RIDAS, Revista Iberoamericana de Aprendizaje Servicio, 12, 104-114. DOI10.1344/RIDAS2021.12.11 
diseño metodológico cualitativo y estructurado en torno al análisis documental y la realización de entrevistas en profundidad a referentes institucionales, graduados, empleadores y autoridades deontológicas de los colegios profesionales. La población objeto estuvo conformada por graduados de la facultad que cursaron sus carreras a partir de la implementación de la PPS, lo cual se configura para la facultad a partir del año 2012 y hasta el año 2018 inclusive. Para la conformación de la muestra se siguió una técnica de muestreo intencional y de muestreo teórico, que aseguró representatividad plural. Como grupo control se empleó una muestra conformada por egresados de promociones anteriores a la implementación de la PPS, empleando el mismo criterio. Con respecto a los referentes externos (informantes claves), los mismos fueron empleadores de ambos grupos de egresados, quienes aportaron una perspectiva sobre el desempeño profesional considerando competencias más allá de lo técnico-disciplinar y referentes de instituciones deontológicas habida cuenta del desempeño ético y social de los graduados necesario de considerar. Se realizó un análisis de contenido a través de una dinámica en espiral de datos provenientes de las diferentes fuentes.

A partir ellos se siguió el enfoque de la teoría fundamentada (Glaser y Strauss, 1967) donde la recolección y análisis de datos se realizan simultáneamente. Las interpretaciones de los datos se sustentaron a través de una codificación sistemática.
Sintéticamente, la investigación se desarrolló en cuatro etapas. En primera instancia se determinaron los alcances deseados en la formación de los graduados de la Facultad de Ciencias Químicas a través de la PPS de la UCC. Para ello, se indagó a través de un análisis documental, el paradigma educativo, la formación y pedagogía en las instituciones de la Compañía de Jesús, así como sus fundamentos y estrategias de implementación de la PPS adoptada durante el período. A partir de ello, se efectuaron entrevistas en profundidad con los responsables de la gestión institucional del período de estudio a los fines de definir las categorías, dimensiones y propiedades de análisis que permitieran abordar el relevamiento con los graduados. Luego, se analizó a través de una muestra problema y un grupo control, las percepciones de los graduados de la Facultad de Ciencias Químicas respecto a la PPS de la universidad y el impacto en su formación. Finalmente, y para lograr consistencia en los resultados, se analizaron las apreciaciones de referentes externos (empleadores y representantes de entidades deontológicas) respecto a la formación y el perfil profesional de los graduados de la Facultad. A modo de síntesis, las dos primeras etapas de la investigación permitieron establecer los efectos de la aplicación de la PPS en dos niveles:

- El modo en que se relaciona la universidad con la comunidad y su entorno, bajo principios de involucramiento activo en experiencias concretas, asociación bidireccional, coproducción de saberes, creación

Giraudo, F. y Gargantini, D. (2021). Revisión de la proyección social institucional a partir del impacto formativo en graduados. RIDAS, Revista Iberoamericana de Aprendizaje Servicio, 12, 104-114. DOI10.1344/RIDAS2021.12.11 
de comunidades de aprendizaje multidisciplinarias, y contribución real hacia los sectores más desfavorecidos.

- Los efectos de la política sobre los futuros graduados de acuerdo al paradigma educativo adoptado. Esta consideración supone la identificación de categorías de análisis y propiedades tanto objetivas como perceptuales referidas:

- al impacto externo o hacia el entorno, con consecuencias a nivel socioambiental y en las políticas públicas;

y al impacto interno o a la transformación interna de la propia persona, con efectos en la formación personal y académico-profesional

Sobre estas categorías teóricas se diseñaron instrumentos que permitieron evaluar la percepción de los graduados de la Facultad acerca del impacto formativo recibido en relación a la PPS, así como la percepción de los referentes externos desde su vínculo con egresados. De su sistematización se extrajeron las principales conclusiones que se exponen.

\section{Resultados y discusión}

Los hallazgos de la investigación acerca del impacto de la PPS en la formación del graduado y las metodologías pedagógicas aplicadas (aprendizajeservicio) en la promoción de experiencias vivenciales de contacto con la realidad bajo el enfoque de RSU adoptado por la UCC, dan cuenta de que la política y experiencias tienen gran influencia particularmente en:

- la comprensión de los conceptos de compromiso social y la promoción de la justicia entendida desde el paradigma formativo promovido por la Compañía de Jesús;

- los impactos a nivel académico que mejoran el aprendizaje;

- la estimulación de una producción científica aplicada;

- la incidencia en políticas públicas;

- el desarrollo de competencias para la inserción profesional de los graduados.

También se han verificado impactos en:

- la generación de estímulos a nivel vocacional;

- la reafirmación de los valores personales y profesionales asumidos;

- el encuentro con la realidad social;

- el reconocimiento y análisis crítico de la realidad, manifestando así alta influencia en las decisiones y participaciones de los graduados en el ámbito personal y profesional. Estos atributos son también rescatados por los 
referentes externos entrevistados como rasgos distintivos del perfil estimulado.

Sin embargo, su desarrollo e impacto cobra sentido y profundidad al combinarse con otros factores destacados como:

- la naturaleza de las propuestas académicas ofrecidas;

- el rol y testimonio de los docentes en el proceso pedagógico estimulado;

- las asignaturas del Área de Formación impartidas (Filosofía, Antropología, Teología y Ética);

- y el ethos institucional experimentado durante los años de formación.

La investigación desarrollada también ha puesto en evidencia la necesidad de cuidar la selección de las experiencias de aprendizaje-servicio promovidas en función de las temáticas y valores que se desean promover (por ejemplo, respeto por la casa común o incidencia en políticas públicas) haciendo expresas dichas opciones. De igual manera se requiere despertar y apoyar el desarrollo de una emociónespiritualidad personal asociada a las experiencias vivenciales que se propongan, a fin de hacerlas significativas más allá de lo técnicodisciplinar o social.

Estas dimensiones y opciones se encuentran estrechamente vinculadas a una espiritualidad institucional, a un modo de vida, costumbres, normas y opciones institucionales que conforman una cultura organizacional y una propuesta formativa específica, coherente con los principios y valores que se quieren promover. En ello existe una relación sinérgica entre experiencias de proyección social y de aprendizaje-servicio, y la consolidación de esta cultura institucional que no debiera soslayarse. Esta retroalimentación constituye la base de la promoción de profesionales altamente formados, conscientes, proféticamente críticos, compasivos y comprometidos con procesos transformadores de las estructuras.

\section{Conclusiones}

Frente a lo expuesto no se encontraron contradicciones entre los hallazgos de la investigación documental acerca del paradigma educativo ignaciano implementado y los diferentes actores involucrados (lo cual da cuenta de una coherencia entre ideario, políticas, procedimientos y resultados alcanzados). Sin embargo, ciertos objetivos institucionales planteados a través de la PPS en la UCC debieran ser revisados a fin de hacer explícitas estas opciones a nivel valórico como instrumental. Entre ellas se destacan algunas vinculadas a dimensiones:

- Conceptuales: la concepción de la PS en términos de transferencia debiera ser superada hacia definiciones explícitas de co-producción y diálogo de saberes donde la naturaleza de los vínculos es central. No se trata solamente de despertar sensibilidades bien intencionadas, sino de introducir 
conceptos basados en un análisis profundo que vincule la realidad y la academia a través de conceptos centrales como ecología integral, fraternidad, cultura democrática y de la paz, derechos humanos, ciudadanía ecológica, inclusión de las diversidades, entre los más notables. De igual manera la reflexión crítica, la incidencia y la necesidad de promover modelos económicos, políticos y culturales alternativos y críticos a los actuales resulta central de incorporar, no como líneas complementarias a la política sino como elementos estratégicos y variables de monitoreo-evaluación en las acciones promovidas.

- Trascendentes/espirituales/ vocacionales: el estímulo explícito de dinámicas motivacionales que permitan delinear, fortalecer y estimular opciones vocacionales vinculadas a opciones trascendentes que superen el desarrollo individual también debieran ser parte explícita de la PPS. El vínculo expreso con las áreas de Formación y Pastoral resulta así un vínculo que lejos de ir en detrimento de la calidad académica, la enriquece y consolida, razón por la cual debiera formar parte de la naturaleza de las experiencias que se promuevan. Junto con ello, el visibilizar perfiles de éxito profesional, docente y científico deseados, mediante testimonios modélicos resulta importante a fin de estimularlos.

- Procedimentales: esta revisión conceptual posee así implicaciones institucionales precisas, como la necesidad de revisar la naturaleza de las convocatorias por proyectos, la asignación de "puntajes" de compromiso social, el fortalecimiento de la articulación investigación-proyección social, el promover espacios de articulación-vinculación actoral para la definición de líneas de trabajo, establecimiento de metodologías de seguimiento de impacto territorial y formativo, promoción no optativa de espacios de reflexión crítica y formación en temas vinculados explícitamente a políticas públicas y al desarrollo de competencias blandas y de liderazgo e incubación de modelos alternativos, debieran formar parte de las líneas operativas de la política.

- Organizacionales: toma así preponderancia el modo de vivir la universidad durante los años de formación (ethos). La vigilancia y manejo adecuado de estos modos, atendiendo a las incoherencias y debilidades éticas más profundas en todas las áreas de actuación resulta fundamental en pos de consolidar una cultura institucional que eduque en torno al modelo de persona y sociedad que se quiere promover.

Estas dimensiones (Figura 1) son las 
que debieran nutrir la PPS vigente a fin de que contribuya realmente en la misión declarada.

Figura 1. Dimensiones ampliadas de la PPS

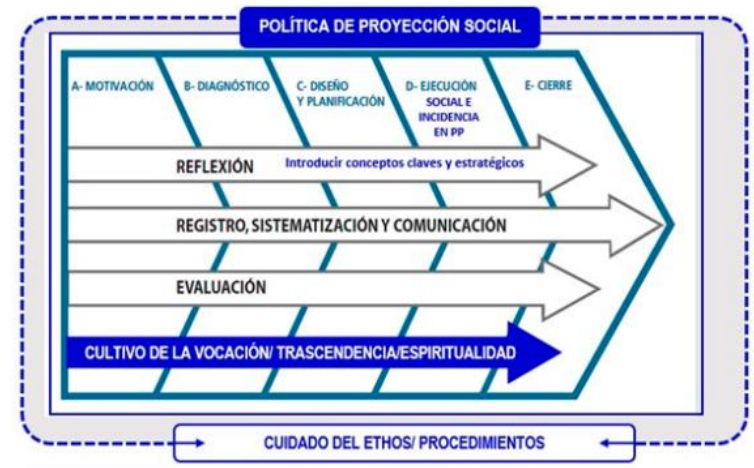

Fuente: Gargantini (2021)

Son ellas los factores genuinos de innovación educativa que debieran distinguirnos por sobre apuestas coyunturales. Sin que ello suceda, las multiplicidades de experiencias de PS y aprendizaje-servicio no dejarán de ser simples placebos que nos impidan como universidades católicas-jesuitas cumplir en profundidad con la misión encomendada.

\section{Referencias bibliográficas}

Aguilar, J.A. (2021). Grandes temas en las Encíclicas Fratelli Tutti y Laudato Si, las preferencias Apostólicas universales de la compañía y los objetivos de desarrollo sostenible. Universidad Javeriana de Cali - CPAL. https://chat.javerianacali.edu.co/sites/ default/files/2021-09/Grandes-Temasen-laudato-si-y-fratelli-tuttiDiagramado.pdf
Álvarez, P. (Ed.). (2014). La Promoción de la Justicia en las Universidades de la Compañía. Promotio Iustitiae, (116).

Secretariado para la Justicia Social y la Ecología.

http://www.sjweb.info/documents/sjs/p $\mathrm{j} /$ docs pdf/pj 116 esp.pdf

Asociación de Universidades Confiadas a la Compañía de Jesús en América Latina [AUSJAL]. (2014). Políticas y Sistema de autoevaluación y gestión de la Responsabilidad Social Universitaria en AUSJAL. Alejandría.

https://www.ausjal.org/documentosinstitucionales/politicas-y-sistema-deautoevaluacion-y-gestion-de-laresponsabilidad-social-universitaria-enausjal

Gargantini, D. (2021, del 28 al 29 de octubre). Espiritualidad institucionalEspiritualidad personal: exigencias de coherencia para una formación transformadora [conferencia]. II Global Simposyum UNISERVITATE.

Aprendizaje-Servicio, Educación Integral y Espiritualidad

Transformadora, Portugal.

Giraudo, F., y Gargantini, D. (2019, del 2 al 3 de septiembre). Propuesta metodológica para evaluar el impacto de la Política de Proyección Social de la UCC en la formación de los graduados de la Facultad de Ciencias Químicas [ponencia]. Congreso Latinoamericano: prácticas, problemáticas y desafíos contemporáneos de la Universidad y del Nivel Superior, Rosario, Argentina.

Glaser, B., y Strauss, A. (1967). El desarrollo de la teoría fundada. Aldine.

Papa Francisco. (20 de febrero de 
2020). Discurso del Santo Padre

Francisco a los participantes en la

Asamblea Plenaria de la Congregación

para la Educación Católica.

https://www.vatican.va/content/frances

co/es/speeches/2020/february/docume

nts/papa-

francesco 20200220 congregaz-

educaz-cattolica.html

Universidad Católica de Córdoba.

(2011). Política relativa a la proyección

social de la Universidad Católica de

Córdoba. Resolución Rectoral N. ${ }^{1} 1092$.

https://www.ucc.edu.ar/portalucc/archi

vos/File/Institucional/2010/DIGESTO U

CC/Vicerrectorado Academico/Secretari

a proyeccion y rsu/Politica-

Proyeccion-Social-UCC(2).pdf 